\title{
A 51-Year Old Woman with Schnitzler Syndrome Treated with Canakinumab
}

\author{
Simon Francis Thomsen ${ }^{*}, 1$, Pernille Hurup Duhn ${ }^{2}$ and Johannes Kristensen ${ }^{1}$ \\ ${ }^{I}$ Department of Dermatology, Bispebjerg Hospital, Copenhagen, Denmark \\ ${ }^{2}$ Department of Rheumatology, Rigshospitalet, Copenhagen, Denmark
}

\begin{abstract}
Schnitzler syndrome is a rare auto-inflammatory disorder characterized by urticarial skin rash, a monoclonal IgM component and at least two of the following findings: periodic fever, arthralgias or arthritis, bone pain, palpable lymph nodes, liver or spleen enlargement, elevated erythrocyte sedimentation rate, leukocytosis, and abnormal findings on bone morphologic investigations. We present the first case of Schnitzler syndrome successfully treated with Canakinumab, a novel anti-IL-1-beta monoclonal antibody.
\end{abstract}

Keywords: Schnitzler syndrome, Canakinumab, Autoinflammatory diseases.

A 51-year old woman with Schnitzler syndrome (SS) diagnosed at the age of 43 presented with a chronic nonpruritic relapsing urticarial rash (Fig. 1), bone pain in the lower legs and arthralgias in the hands, elbows, knees and ankles accompanied by episodic fever and tiredness. Histology of the skin lesions showed urticaria with no signs of vasculitis. There was a monoclonal IgM-kappa component with a highest documented value of $9.87 \mathrm{~g} / \mathrm{l}$. Leucocytes, CRP, cholesterol and ASAT were marginally increased. Three consecutive chest radiographs were normal as was the ECG. She had a previous history of spondylitis in the lumbar spine, enlarged lymph nodes in the retroperitoneum and a positive Mantoux test (but a negative interferon- $\gamma$ release test). However, infection with tuberculosis was never confirmed. She was treated with Isoniazid $300 \mathrm{mg}$ daily for six months prior to treatment with biologics. Past treatments included prednisolone, methotrexate, azathioprin, and rituximab, all of which did not provide sufficient symptom control. For the past four years she had been treated with subcutaneous Anakinra (Kineret) $100 \mathrm{mg}$ once daily as sole treatment except for analgesics and simvastatin. She had a daily consumption of 20 cigarettes.

SS is a rare auto-inflammatory disorder characterized by urticarial skin rash, a monoclonal IgM component and at least two of the following findings: periodic fever, arthralgias or arthritis, bone pain, palpable lymph nodes, liver or spleen enlargement, elevated erythrocyte sedimentation rate, leukocytosis, and abnormal findings on bone morphologic investigations, as long as other known causes for such changes have been excluded [1]. The risk of progression into a lymphoproliferative disorder is $15-20 \%$ and a small proportion of the patients develop AA amyloidosis due to paraproteinemia. To date about a hundred cases of SS have been reported in the literature, most of which are summarized by de Koning et al., [2].

*Address correspondence to this author at the Department of Dermatology, Bispebjerg Hospital, Bispebjerg Bakke 23, DK-2400 Copenhagen NV, Denmark; Tel: +45 2613 9838; Fax: +45 3531 3113; E-mail: sft@city.dk

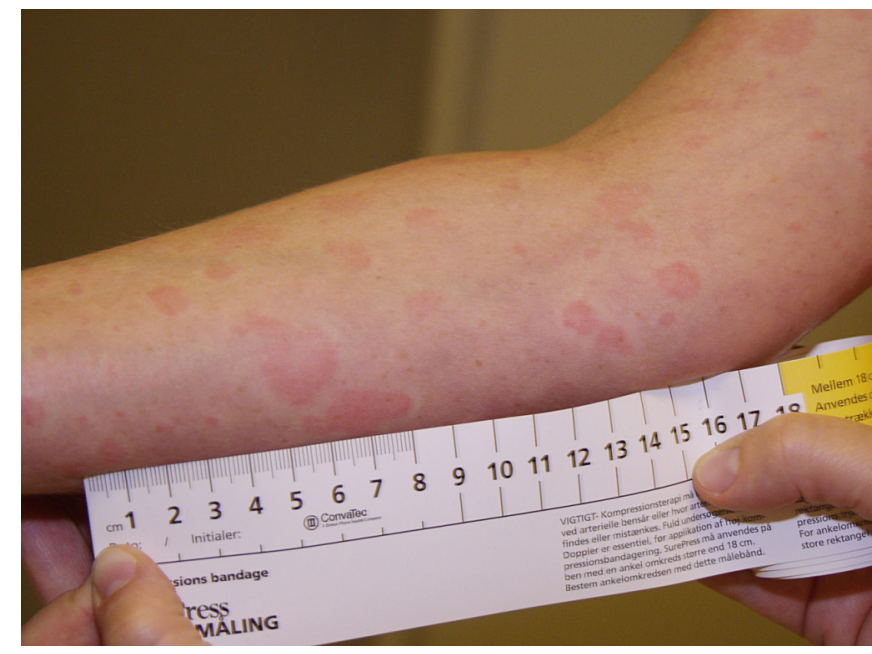

Fig. (1). Urticarial rash in a patient with Schnitzler syndrome.

The IL-1 receptor antagonist Anakinra has shown complete and sustained remission of all symptoms in SS but usually with recurrence of symptoms immediately after discontinuation [1]. Anakinra is administered daily and therefore is a cumbersome treatment. Moreover, injectionsite reactions are common and include erythema, ecchymoses, inflammation, and pain. The level of monoclonal protein does not decrease and neutropenia is a risk, calling for other safe long-term treatment options.

Canakinumab (Ilaris) is a novel and fully human anti-IL1-beta monoclonal antibody. Subcutaneous Canakinumab $(150 \mathrm{mg})$ once every 8 weeks has shown promising results in the treatment of cryoporin associated periodic syndrome (CAPS) and has been approved for this indication by the US Food and Drug Administration and by the European Medicines Agency. Currently, interventional case-series are undertaken for the evaluation of its potential in the treatment of SS [3]. We are unaware of any previous reports in the literature of treatment of SS with Canakinumab.

Our patient was symptomatically fully controlled by Anakinra but due the continuing daily need for 
administration of this drug, which was burdensome to the patient, we begun treatment with Canakinumab twice during a two-week period after discontinuation of Anakinra. During treatment with Canakinumab she experienced a small relapse of bone pain, arthralgias, and a faint rash accompanied by tiredness and suspected fever but had no apparent adverse effects. We observed no harmful (or beneficial) effects of Canakinumab in respect to laboratory data. In particular, the IgM-component was unchanged during the treatment and in fact had increased steadily from 4.60 to $9.87 \mathrm{~g} / 1$ during the entire course of the disease, also while the patient was treated with Anakinra. She scored her symptom relief while treated with Canakinumab as 8 out of 10 on a visual analogue scale compared with 10 out of 10 while treated with Anakinra.
The prolonged duration of action of Canakinumab and possible low frequency of injection-site reactions may confer certain advantages over other IL-1 inhibitors such as Anakinra. Our patient had obvious symptom relief from the treatment with Canakinumab but not as markedly as compared with Anakinra. The role of Cankinumab in the treatment of SS awaits the emergence of further evidence.

\section{REFERENCES}

[1] Lipsker D. The Schnitzler syndrome. Orphanet J Rare Dis 2010; 5: 38.

[2] de Koning HD, Bodar EJ, van der Meer JW, Simon A, Schnitzler Syndrome Study Group. Schnitzler syndrome: beyond the case reports: review and follow-up of 94 patients with an emphasis on prognosis and treatment. Semin Arthritis Rheum 2007; 37(3): 13748.

[3] www.ClinicalTrials.gov (Accessed: Nov 7, 2011). 